\title{
Optical studies of noctilucent clouds in the extreme ultraviolet
}

\author{
J. Hedin ${ }^{1}$, J. Gumbel ${ }^{1}$, M. Khaplanov ${ }^{1}$, G. Witt ${ }^{1,2}$, and J. Stegman ${ }^{1}$ \\ ${ }^{1}$ Department of Meteorology Stockholm University, 10691 Stockholm, Sweden \\ ${ }^{2}$ Department of Earth Sciences, Hebrew University, Jerusalem, Israel
}

Received: 16 January 2007 - Revised: 26 October 2007 - Accepted: 19 December 2007 - Published: 28 May 2008

\begin{abstract}
In order to better understand noctilucent clouds (NLC) and their sensitivity to the variable environment of the polar mesosphere, more needs to be learned about the actual cloud particle population. Optical measurements are today the only means of obtaining information about the size of mesospheric ice particles. In order to efficiently access particle sizes, scattering experiments need to be performed in the Mie scattering regime, thus requiring wavelengths of the order of the particle size. Previous studies of NLC have been performed at wavelengths down to $355 \mathrm{~nm}$ from the ground and down to about $200 \mathrm{~nm}$ from rockets and satellites. However, from these measurements it is not possible to access the smaller particles in the mesospheric ice population. This current lack of knowledge is a major limitation when studying important questions about the nucleation and growth processes governing NLC and related particle phenomena in the mesosphere. We show that NLC measurements in the extreme ultraviolet, in particular using solar Lyman- $\alpha$ radiation at $121.57 \mathrm{~nm}$, are an efficient way to further promote our understanding of NLC particle size distributions. This applies both to global measurements from satellites and to detailed in situ studies from sounding rockets. Here, we present examples from recent rocket-borne studies that demonstrate how ambiguities in the size retrieval at longer wavelengths can be removed by invoking Lyman- $\alpha$. We discuss basic requirements and instrument concepts for future rocket-borne NLC missions. In order for Lyman- $\alpha$ radiation to reach NLC altitudes, high solar elevation and, hence, daytime conditions are needed. Considering the effects of Lyman- $\alpha$ on NLC in general, we argue that the traditional focus of rocket-borne NLC missions on twilight conditions has limited our ability to study the full complexity of the summer mesopause environment.
\end{abstract}

\footnotetext{
Correspondence to: J. Hedin

(jonash@misu.su.se)
}

\section{Introduction}

Ever since the first reports in 1885 (Backhouse, 1885; Leslie, 1885), noctilucent clouds (NLC) have fascinated atmospheric scientists. The clouds form as thin layers at altitudes between 80 and $85 \mathrm{~km}$ in the high-latitude summer mesosphere. It is now widely accepted that they are composed of water ice particles (Hervig et al., 2001; Eremenko et al., 2005) possibly with small nucleation cores. The mesosphere is dry with water concentrations of only a few ppmv (Seele and Hartogh, 1999) and extremely low temperatures are needed for ice particles to nucleate. During the polar summer, mean temperatures as low as $130 \mathrm{~K}$ are reached in the altitude range between 80 and $90 \mathrm{~km}$ (Witt et al., 1964; Lübken, 1999) with local deviations of up to $\pm 15 \mathrm{~K}$ due to wave motions (Philbrick et al., 1984; Rapp et al., 2002). Being extremely sensitive to changes in atmospheric conditions, NLC have recently gained substantial interest as potential indicators of global trends (Thomas et al., 1989; Deland et al., 2003).

Rapp and Thomas (2006) have recently presented a review of mesospheric ice microphysics. Saturation ratios are generally not high enough for homogeneous nucleation, i.e. the spontaneous formation of new particles from a pure gas phase, to be significant. Instead pre-existing ice nuclei are required for the formation of ice particles in the process known as heterogeneous nucleation. Once formed, the ice particles grow by the direct deposition of water vapour onto their surfaces. While the particles consume the available water vapour, gravity makes them settle down. At the same time they are subject to transport by mean winds and small-scale motions, i.e. waves and turbulence. The particles will start to scatter light efficiently such that they may be observed from ground-based or space-borne optical instruments when they reach equivalent spherical radii in excess of about $30 \mathrm{~nm}$.

The size range of visible NLC particles has been studied by optical measurements with satellites (Rusch et al., 1991;

Published by Copernicus Publications on behalf of the European Geosciences Union. 


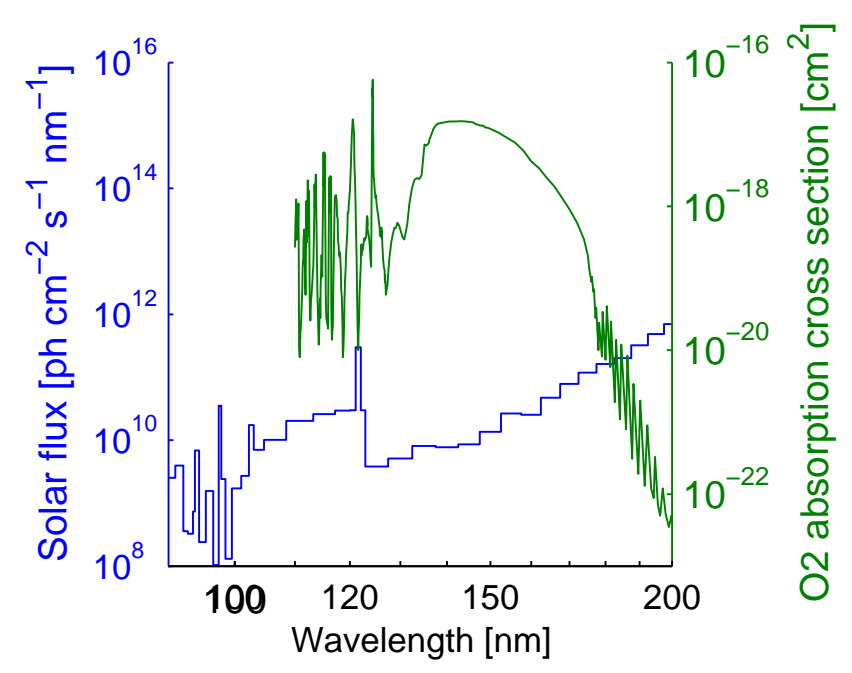

Fig. 1. Solar spectrum and $\mathrm{O}_{2}$ absorption cross section around the Lyman- $\alpha$ line at $121.57 \mathrm{~nm}$.

Merkel et al., 2003; Karlsson and Rapp, 2006), rockets (Gumbel et al., 2001), and lidars (von Cossart et al., 1999; Alpers et al., 2000). These observations show that particle radii generally remain smaller than $100 \mathrm{~nm}$. However, from normal optical measurements it is not possible to access the smaller particles in the mesospheric ice population.

This current lack of knowledge on the smaller particles is a major limitation when studying important questions about the nucleation and growth processes governing NLC and related phenomena in the mesosphere. In particular, nucleation is still poorly understood. During the past four decades, several candidates to the pre-existing nuclei for ice particle formation have been proposed. These include e.g. large hydrated ion clusters (Witt, 1969) and meteoric material (Rosinski and Snow, 1961; Hunten et al., 1980; Plane, 2000). The existence of large hydrated ion clusters in the low-temperature environment is well known. However, because of dissociative recombination with electrons it has been doubted that ion clusters can efficiently grow to the critical size of condensation (Kopp et al., 1985). There is today indirect experimental evidence for the existence of nanometre sized meteoric smoke particles (Rapp et al., 2007a), which have long been considered the most likely candidate. Closely connected to nucleation and growth are open questions about the NLC particle size distribution. In general, the analysis of optical NLC data needs to be based on assumptions on a mathematical form of the size distribution in the observed particle population. A size retrieval can then fit a limited number of parameters, e.g. an effective radius and a width, to this distribution. Early analysis of the optical properties of NLC particles was restricted to the power-law distribution sufficient to describe the overlap of the scattering function with the size distribution (Witt, 1968). Many later satellite studies have assumed lognormal size dis-

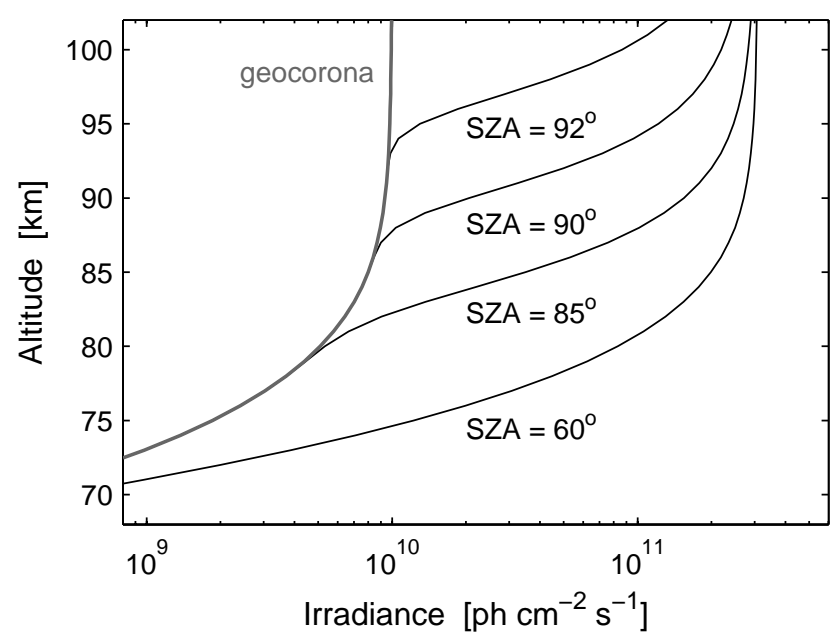

Fig. 2. The penetration of direct and geocoronal Lyman- $\alpha$ into the mesosphere. The geocoronal contribution (grey line) is almost independent of the solar position and dominates at typical NLC altitudes for solar zenith angles greater than $90^{\circ}$.

tributions (e.g. Thomas and McKay, 1985; von Savigny et al., 2005). Also ground-based lidar observations at three or more wavelengths have been analysed based on this assumption (von Cossart, 1999; Alpers et al., 2000). This form of distribution would be appropriate to an aged coagulated aerosol (Friedlander, 1961) but its application to noctilucent clouds can only be justified by computational convenience. Rapp and Thomas (2006) found that their modelled ice particle size distributions were closely described by a Gaussian distribution, whereas the use of a lognormal distribution lead to a severe overestimate of the abundance of large particles. Berger and von Zahn (2002) also predicted size distributions closely described by a Gaussian shape. Comparing the microphysical model results from Rapp and Thomas (2006) to lidar measurements and satellite-borne NLC observations, Rapp et al. (2007b) found consistency between Gaussian size distributions and the available experimental data under the condition of needle- or plate-like spheroid-shaped particles. None of the considered data sets were consistent with the assumption of spherical particles.

Optical measurements are today the only means of obtaining information about the size of NLC particles. New information about the properties of the particles has become available from measurements in the infrared (Hervig et al., 2001; Eremenko et al., 2005). However, in order to access particle sizes efficiently, the deviation of the particle's scattering/absorption properties from the pure Rayleigh regime needs to be studied. This requires wavelengths of the order of the particle size and, hence, measurements in the visible and, in particular, the ultraviolet part of the spectrum. Previous NLC measurements have been made at wavelengths down to $355 \mathrm{~nm}$ with ground-based instruments (e.g. von Cossart et al., 1999) and down to about $200 \mathrm{~nm}$ with rockets and 
satellites (e.g. Rusch et al., 1991; Gumbel et al., 2001). In this paper we argue that measurements at even shorter wavelengths in the extreme ultraviolet (EUV) are necessary to extend our knowledge of the NLC particle size distribution. In particular, Lyman- $\alpha$ radiation at $121.57 \mathrm{~nm}$ is well suited for such studies as NLC are well illuminated by solar Lyman- $\alpha$ during daytime conditions. At $121.57 \mathrm{~nm}$ even small mesospheric ice particles come well into the Mie scattering regime and start to influence the total NLC scattering. Therefore, an investigation of the scattering and extinction properties of ice particles at Lyman- $\alpha$ can yield unique and important complementary information about the polar mesospheric ice layer.

In Sect. 2 we give a general description of Lyman- $\alpha$ radiation in the atmosphere. Section 3 discusses in more detail the scattering of Lyman- $\alpha$ by mesospheric particles and shows how NLC properties can be retrieved. In Sect. 4 we present the instruments and basic requirements for such measurements. We conclude with an outlook on sounding rocket projects in Sect. 5.

\section{Lyman- $\alpha$ in the mesosphere}

The ${ }^{2} \mathrm{P}-{ }^{2} \mathrm{~S}$ resonance transition of atomic hydrogen is the strongest and most conspicuous emission feature of the solar EUV spectrum (Meier, 1991). Due to a favourable coincidence, the wavelength of the Lyman- $\alpha$ line of $121.57 \mathrm{~nm}$ coincides with a minimum of the $\mathrm{O}_{2}$ absorption spectrum (Fig. 1) that efficiently shields the mesosphere from other ultraviolet radiation in the wavelength range $100-180 \mathrm{~nm}$. Thus, direct Lyman- $\alpha$ radiation penetrates well into the mesosphere. The interaction of the Lyman- $\alpha$ radiation with atmospheric constituents has two main effects. First, the radiation can ionize the nitric oxide molecule, which gives rise to the ionospheric D-layer. Even more important, photolysis by Lyman- $\alpha$ is the main loss process for water vapour (Bates and Nicolet, 1965; Lewis et al., 1983). The extinction of direct solar Lyman- $\alpha$ is actually a measure of the $\mathrm{O}_{2}$ column density and has been used to determine the atmospheric temperature profile from sounding rockets (Thrane and Johannessen, 1975; Prinz and Brueckner, 1977).

In addition to the direct solar illumination, the mesosphere is illuminated by solar Lyman- $\alpha$ radiation resonantly scattered by hydrogen atoms that have escaped from the Earth's atmosphere and constitute the geocorona (Meier and Mange, $1970 ; 1973)$. This diffuse radiation is observable both in daytime and with the Sun below the horizon. For detailed radiative transfer simulations it is important to note that this multiply scattered Lyman- $\alpha$ line is significantly narrower than the Lyman- $\alpha$ line incident from the sun (Meier, 1991). In the mesosphere, the Lyman- $\alpha$ radiation field also depends on resonant scattering by the local hydrogen atom distribution below $100 \mathrm{~km}$. This in turn is determined by photo-dissociation of $\mathrm{H}_{2} \mathrm{O}$ and the catalytic chemical reaction scheme of mesospheric odd hydrogen.
Figure 2 shows a model calculation of the penetration of direct and geocoronal Lyman- $\alpha$ into the mesosphere. The direct flux is calculated with a spherical model using a high-latitude July atmosphere from MSIS-90e (Hedin, 1991). During the solar cycle the direct Lyman- $\alpha$ irradiance at the top of the atmosphere varies typically between $2 \times 10^{11}$ and $4 \times 10^{11} \mathrm{ph} \mathrm{cm}^{-2} \mathrm{~s}^{-1}$ (Lean, 1987), we have chosen $3 \times 10^{11} \mathrm{ph} \mathrm{cm}^{-2} \mathrm{~s}^{-1}$ here. In a detailed radiative transfer calculation, the temperature-dependence of the $\mathrm{O}_{2}$ absorption and its wavelength dependence in the vicinity of the Lyman- $\alpha$ line need to be taken into account (Carver et al., 1977). For the general estimate in Fig. 2, we use a constant absorption cross section of $1.0 \times 10^{-20} \mathrm{~cm}^{2}$. For the calculation of the geocoronal Lyman- $\alpha$ flux, we assume an isotropic radiance field of $10^{10} / \pi \mathrm{ph} \mathrm{cm}^{-2} \mathrm{~s}^{-1} \mathrm{str}^{-1}$ at the top of the atmosphere (Meier and Mange, 1973). Using the spherical model, radiances from different zenith directions are than propagated through the atmosphere and integrated over the different directions in order to obtain the irradiance at a given altitude. Figure 2 presents the resulting geocoronal irradiance of a horizontally oriented surface.

Figure 2 shows a strong dependence of the direct Lyman- $\alpha$ flux on the solar zenith angle (SZA). In particular, for twilight conditions $\left(\mathrm{SZA} \approx 90^{\circ}\right.$ ) there is a strong dependence in the altitude range of interest for mesospheric ice particles, $80-90 \mathrm{~km}$. For solar zenith angles larger than $90^{\circ}$, the diffuse geocoronal flux dominates over the direct flux at these altitudes. It is important to note that many experimental studies of NLC have been made only during these twilight conditions (e.g. Goldberg et al., 1993; 2001).

The presence of ice particles in the summer mesopause region between 80 and $90 \mathrm{~km}$ is itself a factor that influences the propagation of Lyman- $\alpha$ radiation. At the wavelength $121.57 \mathrm{~nm}$ ice has a substantial absorption owing to a complex refractive index, at room temperature $\tilde{n}=1.3963-0.2470 i$ (Warren, 1984). If the solar radiation at Lyman- $\alpha$ penetrates down to the NLC layer, the ice particles in the cloud scatter this radiation. On the dayside with solar zenith angles less than $\sim 90^{\circ}$, Lyman- $\alpha$ scattering by ice particles is dominated by the direct solar flux outside the narrow geocoronal resonance line (Fig. 2) and thus independent of the detailed atmospheric distribution of atomic hydrogen. At $85^{\circ} \mathrm{SZA}$ atmospheric extinction is almost two orders of magnitude at the NLC altitude, whereas at $60^{\circ}$ the flux is only a factor of three smaller than at the top of the atmosphere. Under these conditions, the scattered Lyman- $\alpha$ from NLC appears as an enhancement from a thin altitude layer that can be observed in a sounding rocket measurement. 

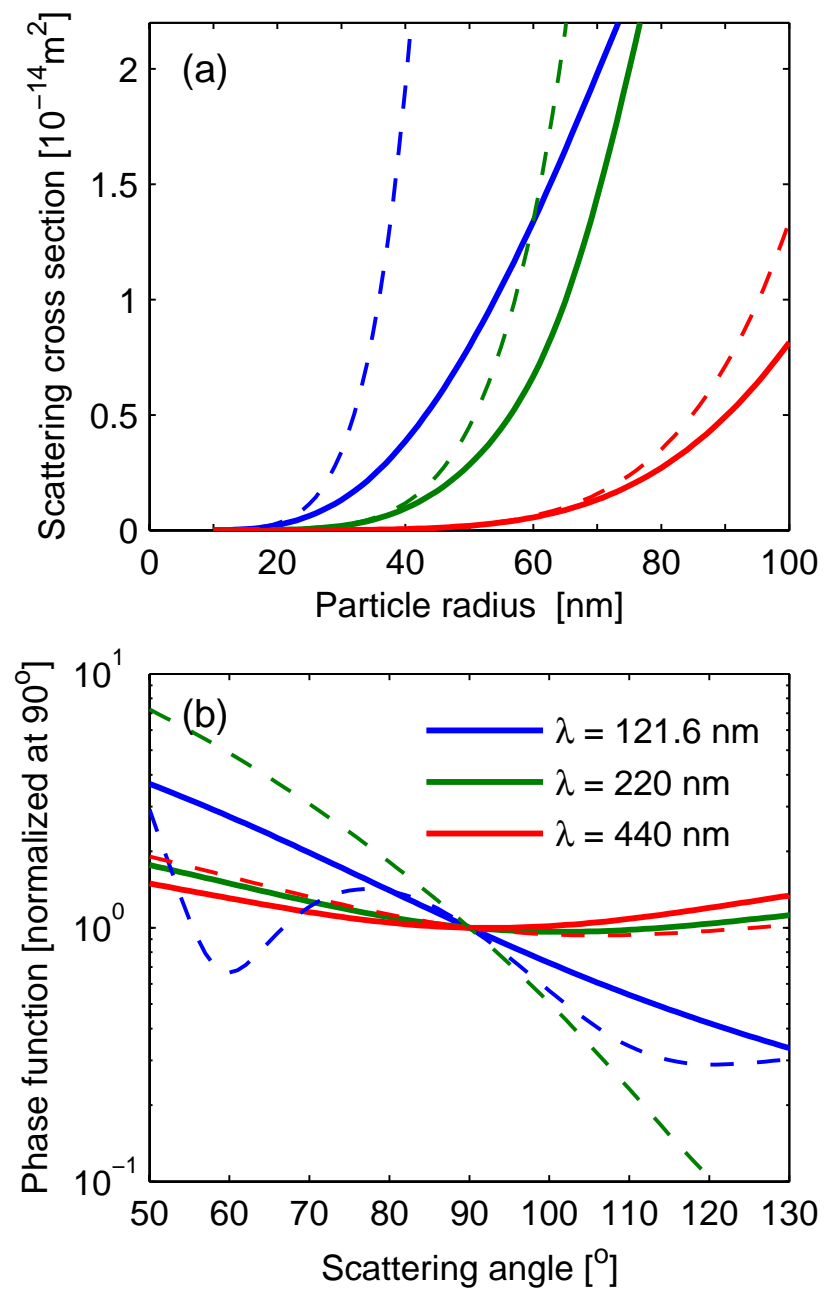

Fig. 3. (a) Mie scattering cross section for spherical ice particles at different wavelengths (solid lines) and comparison to the $r^{6}$ dependence of pure Rayleigh scattering (dashed lines). (b) Phase functions at different wavelengths for particles of radii $30 \mathrm{~nm}$ (solid lines) and $70 \mathrm{~nm}$ (dashed lines). The colour code is the same for both panels with blue, green and red lines representing Lyman- $\alpha$, $220 \mathrm{~nm}$ and $440 \mathrm{~nm}$, respectively.

\section{Retrieval of NLC properties}

\subsection{Scattering by mesospheric ice particles}

The basic idea of NLC photometry is to relate optical characteristics of the cloud to properties of the cloud particle population. Four pieces of information are in principle available from photometric NLC measurements: the total scattered radiance, spectral dependence of the scattering (colour ratio, if two or more wavelengths are measured), angular dependence of the scattering (the phase function, if the photometer is tilted with respect to the spin axis of the payload), and polarisation properties of the scattered light (if polarisers are used). The retrieval of particle properties from these optical
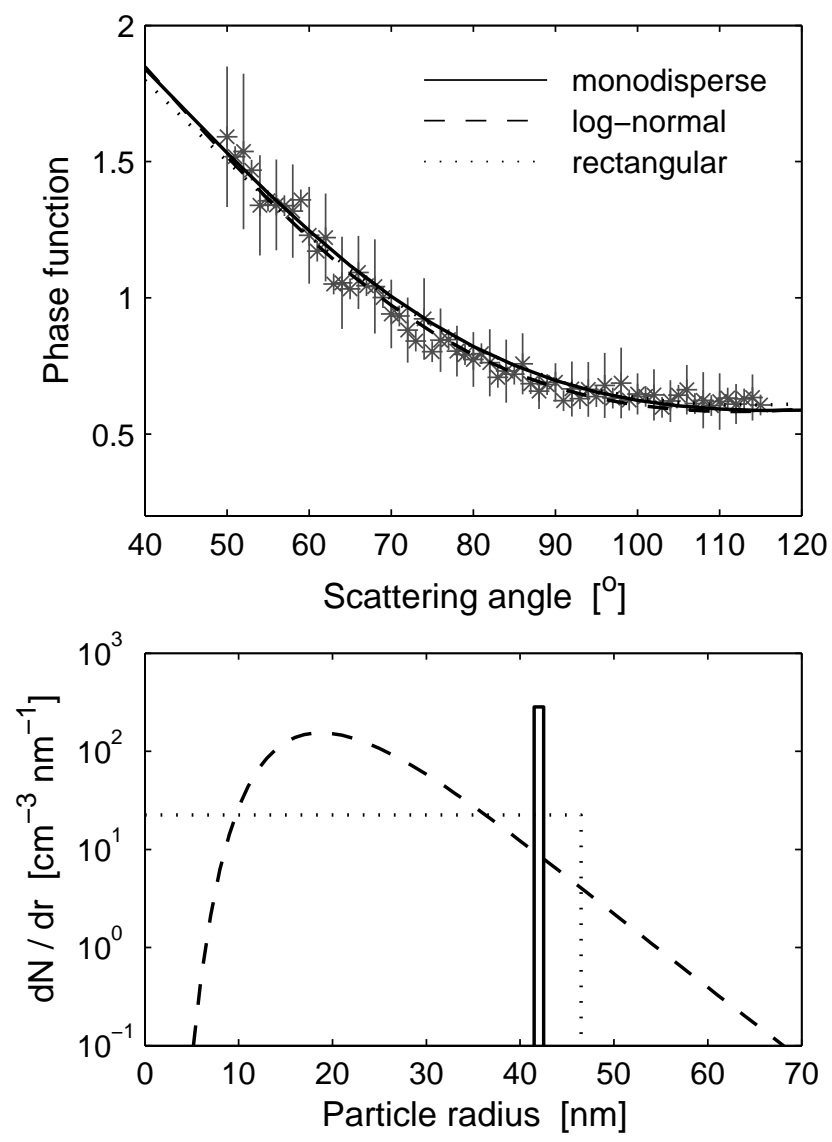

Fig. 4. An example of the ambiguity of photometric NLC measurements. Upper panel, points: Phase function of an NLC measured during the DROPPS-2 rocket flight at wavelength $222 \mathrm{~nm}$ (Gumbel et al., 2001). Lines: Calculated phase functions that all fit the measurement and that correspond to the three different NLC particle populations in the lower panel (Gumbel and Witt, 2001). Lower panel: Particle populations representing a monodisperse distribution $(r=42 \mathrm{~nm})$, a rectangular distribution $\left(r_{\max }=46 \mathrm{~nm}\right)$ and a lognormal distribution $\left(\sigma=1.4, r_{\text {mod }}=21 \mathrm{~nm}\right)$.

data requires a theoretical description of the scattering process and assumptions about the particle populations. Particle properties are then obtained by comparing the measured scattering parameters to simulation results from Mie calculations or other theoretical approaches. In this way, one or more size parameters can be fitted to an assumed size distribution. Phase function, colour ratio and polarisation are generally functions of the size and shape of the particles as well as the wavelength of the incident light. In Fig. 3a the Mie scattering cross section is plotted as a function of particle radius for spherical ice particles at the three wavelengths Lyman- $\alpha, 220 \mathrm{~nm}$ and $440 \mathrm{~nm}$. The figure demonstrates that the scattering properties at Lyman- $\alpha$ deviate from pure Rayleigh scattering for NLC particles as small as $15 \mathrm{~nm}$. At visible wavelengths, on the other hand, the scattering by typical NLC particles is close to the Rayleigh regime with 


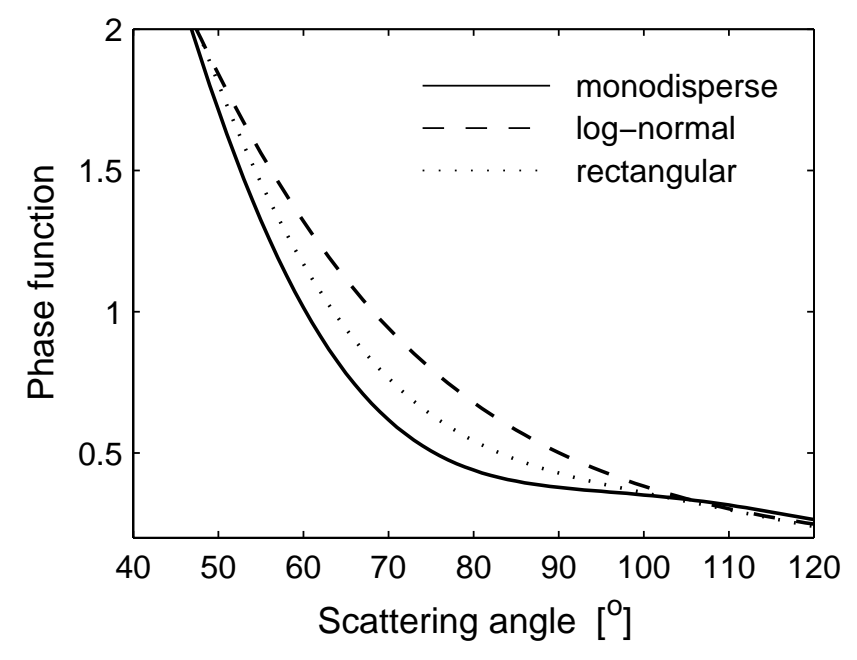

Fig. 5. Phase functions corresponding to the particle populations in Fig. 4, now calculated for Lyman- $\alpha$. By studying the phase functions at $222 \mathrm{~nm}$ and Lyman- $\alpha$, the ambiguity in the optical analysis is removed.

the cross section $\sigma_{s}$ increasing nearly with $r^{6}$ (e.g. Bohren and Huffman, 1983),

$\sigma_{s}=\frac{128 \pi^{5} r^{6}}{3 \lambda^{4}}\left(\frac{n-1}{n+2}\right)^{2}$

where $n$ is the refractive index. This strong size dependence means that the few largest particles in a population completely dominate the optical properties of NLC. Hence, it is not possible with optical measurements in the visible and near UV to draw conclusions about the smaller particles in a distribution. Measurements in the EUV open this possibility. Figure $3 \mathrm{~b}$ shows part of the phase functions for particle radii 30 and $70 \mathrm{~nm}$ at the above three wavelengths. The Rayleigh phase function is symmetric between forward and backward scattering. Again, as opposed to the longer wavelengths, the scattering at Lyman- $\alpha$ deviates significantly from this symmetric Rayleigh phase function even for particles of $30 \mathrm{~nm}$ radius.

In the following, we present examples from rocket-borne NLC photometry at 220 and $440 \mathrm{~nm}$. We then show what additional information can be obtained by adding a measurement channel at Lyman- $\alpha$.

\subsection{Visible and near-UV photometry}

Rocket-borne optical measurements of NLC are usually performed with photometers in an upward viewing direction in order to avoid the bright background radiation from below. A photometer viewing in an upward direction detects sunlight scattered from the molecular column and, if present, from an overhead NLC layer. As the payload ascends, the radiance detected from the molecular Rayleigh scattering decreases monotonically with altitude while the contribution from the

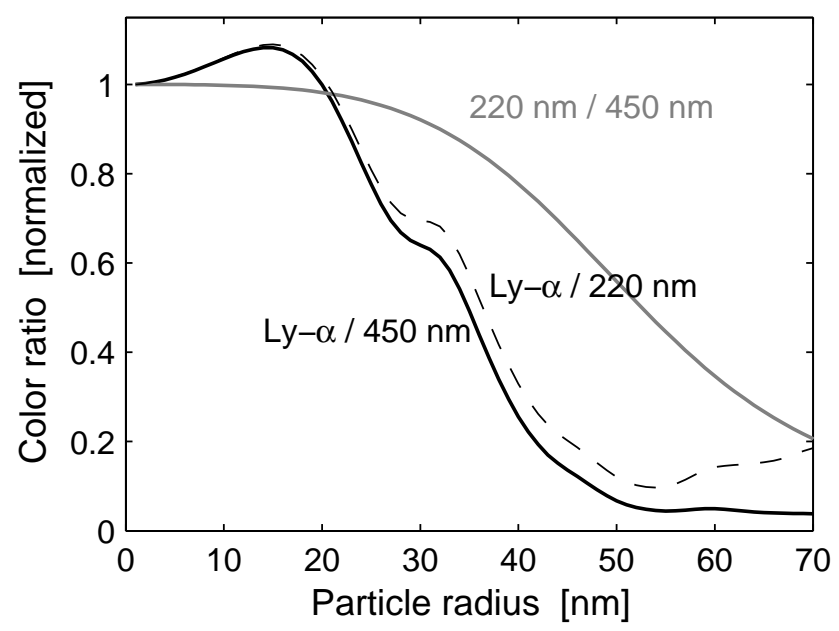

Fig. 6. Ratios in the scattering efficiency at Lyman- $\alpha, 220 \mathrm{~nm}$ and $450 \mathrm{~nm}$. The ratios apply to a scattering angle of $90^{\circ}$ and are normalized to Rayleigh scattering of the smallest particles. Significant deviations from Rayleigh scattering start at particle radii around $25 \mathrm{~nm}$ when measurements at Lyman- $\alpha$ are invoked.

scattering NLC layer remains essentially constant until the NLC layer is reached. The passage through the NLC is then marked by a sharp decrease of the detected radiance, and the exact altitude profile of the NLC is thus readily detected insitu (Witt et al., 1975). Above the NLC layer only weak molecular scattering and possibly other upper atmospheric background sources are left. The known molecular Rayleigh scattering can be utilized for absolute calibration of the NLC radiance. In addition, a photometer fitted with a polarisation filter on a rotating rocket will produce a sinusoidally modulated signal, which allows the recovery of the three Stokes parameters I, Q, and U of the scattered radiation. A complication for measurements at longer wavelengths is that upwelling light scattered from the ground or from the lower atmosphere also contributes to the illumination of the NLC. If optical measurements are extended to the UV, the background from below is removed since wavelengths shorter than $300 \mathrm{~nm}$ are completely absorbed by stratospheric ozone. This, in addition to the possibility of measuring deeper into the Mie scattering regime, is another major advantage of NLC photometry at shorter wavelengths.

An example of rocket-borne photometric NLC studies is the MIDAS-DROPPS campaign from Andøya, Norway, in 1999 (Goldberg et al., 2001). On two DROPPS payloads, photometers viewing $30^{\circ}$ from the rocket spin axis scanned the overhead NLC layer with scattering angles of $50-115^{\circ}$ and thus obtained the scattering phase function of the NLC particle population. This is shown in Fig. 4 for the photometer measurements at $222 \mathrm{~nm}$ wavelength. Gumbel et al. (2001) analysed this phase function using a Mie calculation and determined an effective optical particle radius of $42 \mathrm{~nm}$ for this cloud. While this determines the size range 


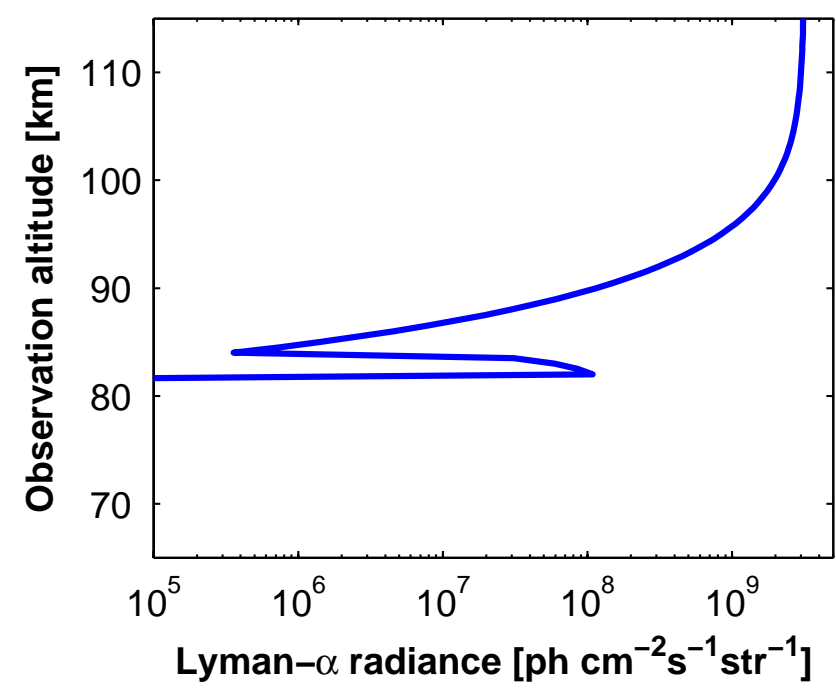

Fig. 7. Simulation of the radiance detected from the geocorona and an NLC for a rocket-borne Lyman- $\alpha$ detector viewing horizontally. The NLC was modelled as a cloud of thickness $2 \mathrm{~km}$ with $100 \mathrm{~cm}^{-3}$ particles of radius $50 \mathrm{~nm}$.

of the large particles that dominate the optical properties of the NLC, Gumbel and Witt (2001) noted that nothing can be concluded about the smaller particles in the population. This ambiguity in the optical measurement is demonstrated in Fig. 4. In addition to the monodisperse particle radius of $42 \mathrm{~nm}$, a lognormal distribution with appropriate mode radii and a rectangular distribution with appropriate cut-off radius are plotted. All fit the measured phase function equally well.

Gumbel and Witt (2001) also investigated whether this ambiguity can be removed by analysing additional scattering information like the spectral dependence. To this end, they calculate the colour ratio between NLC measurements at $222 \mathrm{~nm}$ and $449 \mathrm{~nm}$ onboard DROPPS. They find that phase function result and colour ratio result are consistent for all three distributions, and, hence, that phase function and colour ratio are redundant and do not provide complementary size information on the particle population.

\subsection{Lyman- $\alpha$ photometry}

The situation changes when measurements at EUV wavelengths are included. Fig. 5 shows the phase functions for the particle populations in Fig. 4 re-calculated for Lyman$\alpha$. The ambiguity in the analysis is now removed. As the shorter Lyman- $\alpha$ wavelength pushes the scattering into the Mie regime the phase function is more dominated by forward scattering. Hence, as shown in Fig. 5, the scattering at angles larger than $60^{\circ}$ decreases compared to the $222 \mathrm{~nm}$ case. In particular, the fewer small particles a distribution contains, the more it deviates from the Rayleigh regime and the more the scattering is reduced at the angles encountered during the rocket flight. Hence, by combining the scattering results at $222 \mathrm{~nm}$ and at Lyman- $\alpha$, it becomes possible to distinguish the different size distributions and to draw conclusions about the abundance of smaller particles in the NLC population. Figure 6 demonstrates the possibility of accessing smaller particles for the spectral analysis. The near-UV colour ratio studied on the DROPPS payloads does not deviate significantly from the Rayleigh scattering ratio for particles smaller than about $40 \mathrm{~nm}$. This limit is reached around $25 \mathrm{~nm}$ for colour ratios with Lyman- $\alpha$.

The magnitude of the scattered Lyman- $\alpha$ signal can be readily estimated relative to the detected signal at $222 \mathrm{~nm}$. The DROPPS $222 \mathrm{~nm}$ photometer used a filter bandwidth of $10 \mathrm{~nm}$. The solar flux in the Lyman- $\alpha$ line is about two orders of magnitude less than the solar flux in a $10 \mathrm{~nm}$ band around $222 \mathrm{~nm}$. Applying Mie calculations to an NLC particle of $40 \mathrm{~nm}$ radius and a scattering angle of $90^{\circ}$, the scattering cross section at Lyman- $\alpha$ is about one order of magnitude larger than the scattering cross section at $222 \mathrm{~nm}$. Hence, the overall NLC signal expected from Lyman- $\alpha$ scattering is typically one order of magnitude less than that detected with a photometer at $222 \mathrm{~nm}$.

In the present work we have not considered studies of the polarisation properties of Lyman- $\alpha$ scattering by NLC. Nevertheless, we believe that such measurements possible with polarisation detectors that use a Wollaston or Rochon prism.

\section{Instruments}

\subsection{Basic requirements}

In order to take maximum advantage of the additional scattering information available in the EUV, a suitable Lyman- $\alpha$ detector should be combined on a sounding rocket with NLC photometers that operate at longer wavelengths. While at longer wavelengths the major source of the disturbing background is scattered radiation from lower altitudes, the critical background for Lyman- $\alpha$ photometry is the diffuse geocorona from above. Hence, measurements of Lyman- $\alpha$ radiation scattered on NLC particles are preferably performed with the optical instrument viewing downward. Figure 7 shows a simulation of the limiting case of a Lyman- $\alpha$ detector viewing horizontally. The NLC was modelled as a cloud of $2 \mathrm{~km}$ thickness with 100 particles $\mathrm{cm}^{-3}$ of radius $50 \mathrm{~nm}$. The solar zenith angle determining the Lyman- $\alpha$ illumination is $60^{\circ}$ and the scattering angle is $90^{\circ}$. The figure shows that the geocoronal background in the $80-90 \mathrm{~km}$ range is comparable to the scattered signal expected from NLC. On the other hand, background from molecular (Rayleigh) scattering by the atmosphere at Lyman- $\alpha$ is completely negligible. At longer wavelengths, Rayleigh scattering from the background atmosphere below about $60 \mathrm{~km}$ becomes comparable to typical NLC brightnesses (Rusch et al., 1991). At Lyman$\alpha$, Rayleigh scattering from these low altitudes is completely suppressed due to the absorption by $\mathrm{O}_{2}$.

Given that the geocoronal background prevents us from looking upward, there are two options: the rocket instrument 
can look tangentially through the cloud or downward. The tangential viewing geometry has the big advantage that the very long line-of-sight through the NLC provides a much stronger NLC signal. Nevertheless, we choose the downward viewing geometry for several reasons: 1 ) The very idea of the rocket-borne measurement is to provide local data that can be directly compared to other in situ experiments on the rocket. By viewing tangentially, the observation path through the cloud can be up to several $100 \mathrm{~km}$ and, hence, the locality of the measurement gets lost. 2) When looking tangentially, the exact path through the NLC is extremely sensitive to the exact payload attitude. Given the limitations of the attitude information typically available from sounding rocket flights, it is difficult to obtain quantitative measurements with tangential geometries since the exact observation path through the cloud layer remains uncertain. 3) When viewing tangentially, the observation period is limited to the actual passage of the NLC layer, thus reducing the available measurement time to a few seconds only. 4) A basic idea of our experiment is to view the NLC layer under different angles in order to obtain information about the scattering phase function. This is best done using a tilted photometer on a spinning payload.

What scattering angles are accessible with typical instrument and flight geometries? The payload does not fly vertically, but points into a zenith direction of typically $60-70^{\circ}$ in the mesosphere. A typical instrument is tilted $30^{\circ}$ with respect to the payload symmetry axis. This means that this instrument covers scattering angles of typically $60-120^{\circ}$ if the solar zenith angle is $60^{\circ}$ and the payload is flying away from the sun. Data at forward scattering angles smaller than $60^{\circ}$ would in principle be desirable. However, for typical rocket photometers stray light in the baffle system tends to become a problem for scattering angles smaller than $60^{\circ}$. Hence, we expect the best data by focusing our approach on scattering angles larger than $60^{\circ}$. For optical measurements in the atmosphere it is desirable to have an instrument with good selectivity and high throughput of the wanted signal (spectral line or interval) but at the same time a good blocking of the radiation at longer and shorter wavelengths. For the current application this means that the instrument should discriminate Lyman- $\alpha$ from other EUV radiation and ideally be completely "solar blind" at wavelengths longer than $160 \mathrm{~nm}$. Solar blindness at these longer wavelengths can be achieved by choosing detectors with suitable cathode material, e.g. KBr. The discrimination against other EUV radiation can be achieved with grating instruments or filter photometers. At UV wavelengths the advantages of filter photometers are lost because of material properties and holographic concave diffraction gratings are preferable. If measurements of the scattering phase function are desired, it is also necessary to view at different angles. Finally, the instrument should be small and easy to incorporate into a given payload structure. Two different rocket instruments for Lyman- $\alpha$ are described below.

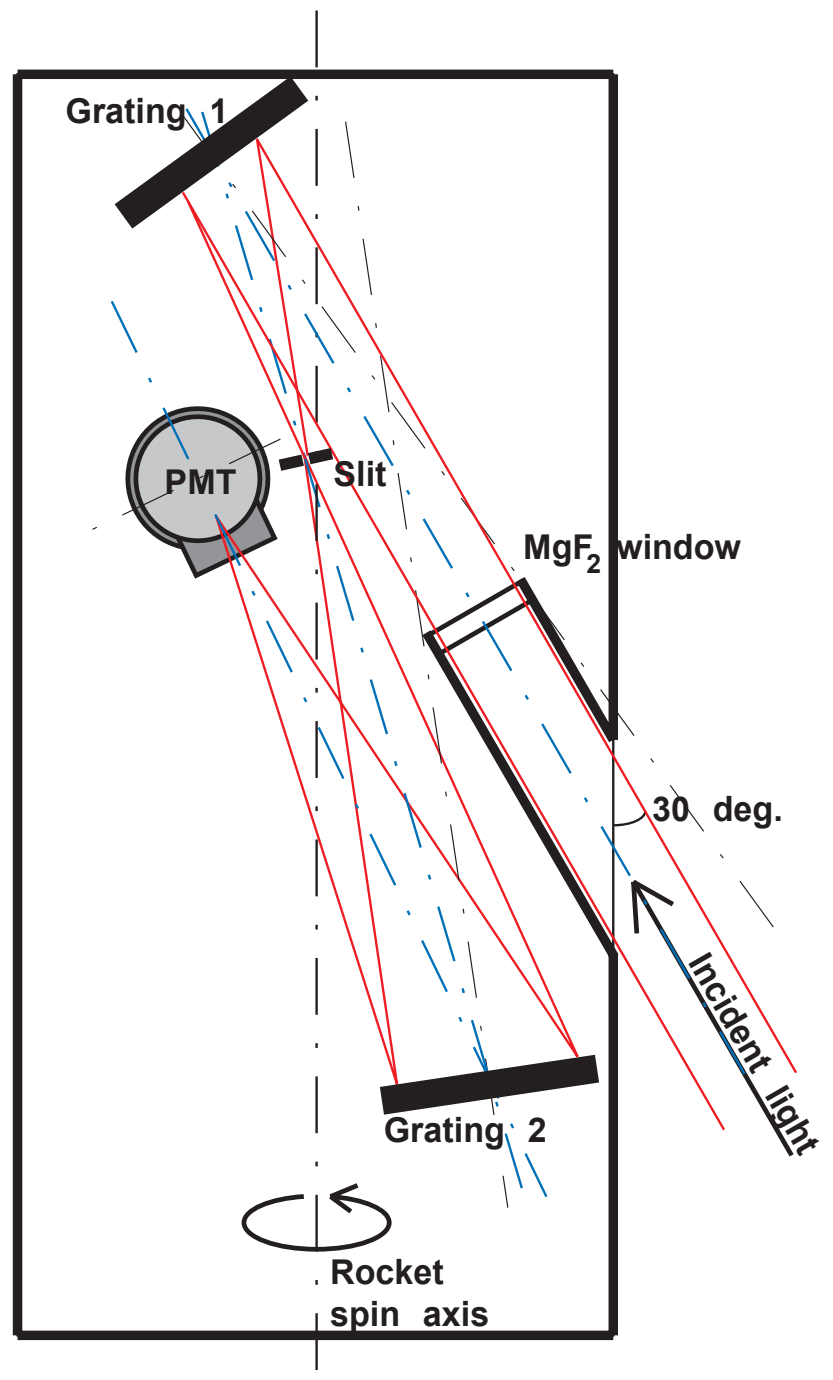

Fig. 8. Schematic of the SLAM grating instrument. The instrument is downward viewing with the opening tilted $30^{\circ}$ from the rocket spin axis. Light enters through a $\mathrm{MgF}_{2}$ window and is reflected on the first grating. A slit transmits the -1 order diffracted light to the second grating. The CsI photomultiplier tube (PMT) is positioned to receive the -1 order diffracted light from the second grating. The entrance aperture is $4 \mathrm{~cm}^{2}$ and the field-of-view is $2^{\circ}$ by $10^{\circ}$, or $6.1 \times 10^{-3}$ steradians.

\subsection{A grating instrument}

The SLAM instrument (Scattered Lyman-Alpha in the Mesosphere) is a new development and a test of the measurement concept. The instrument is a double monochromator using a 3600 lines/mm holographic grating in a Woolsworth-type mounting as collimator, a second grating, identical to the first, in a Rowland circle mounting as dispersive element, and a CsI side-on photomultiplier as detector (Fig. 8). The position and orientation of the gratings were calculated to select the Lyman- $\alpha$ line. This setup provides a blocking by 


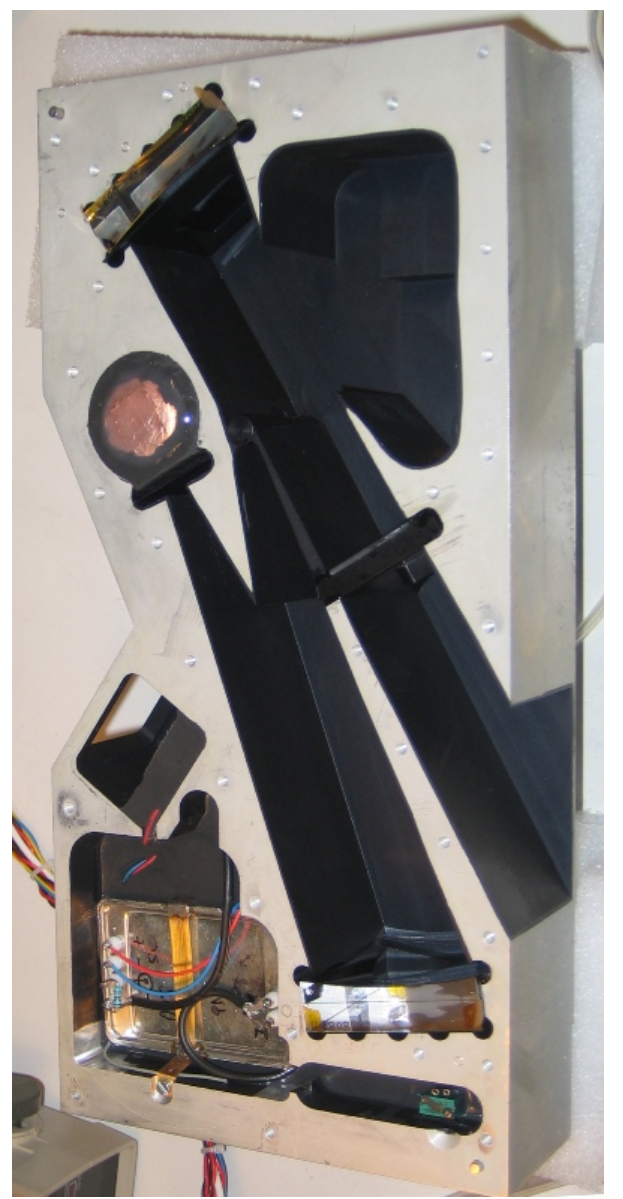

Fig. 9. The open SLAM instrument (without the cover plate) complete with gratings, photomultiplier tube, and electronics, the entrance is to the right and an additional sun sensor is to the left.

6-8 orders of magnitude at other wavelengths. A limitation of this instrument is its sensitivity. The two gratings together transmit only $\sim 3 \%$ of the selected wavelength and the quantum efficiency of the photomultiplier is $\sim 25 \%$ at $121.57 \mathrm{~nm}$. Hence, over two orders of magnitude of the signal are lost in the instrument.

As for the geometry of SLAM, the instrument is designed to look downward at an angle of $30^{\circ}$ from the rocket spin axis. It is machined from a solid aluminium block containing both optical setup and electronics components (Fig. 9). The inside walls are electrochemically blackened, a cover plate and an $\mathrm{MgF}_{2}$ window make the instrument vacuum tight. The solid construction provides the instrument with a large heat capacity. Together with the fact that the nosecone protects SLAM from aerodynamic heating until $60 \mathrm{~km}$ altitude, this ensures a constant instrument temperature during the mesospheric measurement. As water vapour is a strong absorber for Lyman- $\alpha$, long-term water desorption from the walls inside the instrument is a major challenge. In order to minimise this effects, SLAM is filled with $1 \mathrm{~atm}$ dry nitro-

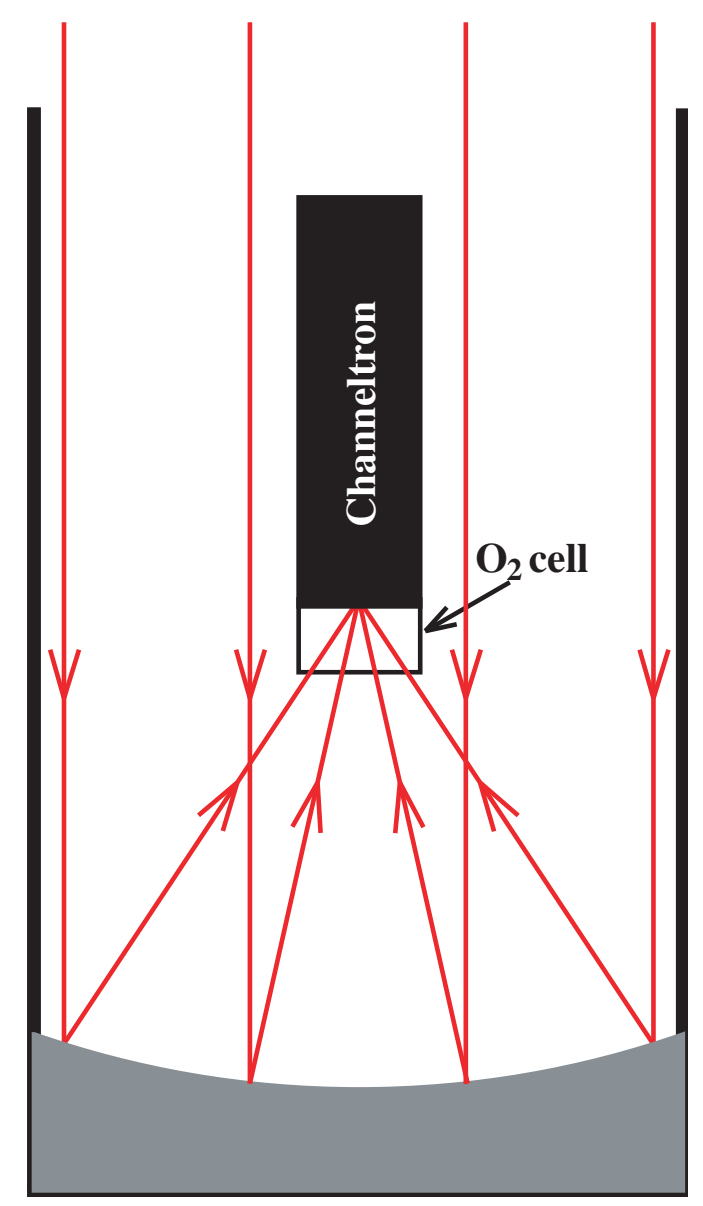

Fig. 10. Schematic of a filter photometer for the measurement of Lyman- $\alpha$. The design is based on a Cassegrain setup with a channel electron multiplier (channeltron) as detector. After the mirror the light enters an $\mathrm{O}_{2}$ cell which absorbs radiation up to $180 \mathrm{~nm}$ except the Lyman- $\alpha$ line at $121.57 \mathrm{~nm}$. A channeltron with a $\mathrm{KBr}$ coated photocathode blocks wavelengths longer than $160 \mathrm{~nm}$.

gen during flight preparation. After releasing the nosecone at $60 \mathrm{~km}$, the instrument is opened by means of a venting hole. In this way, the instrument is efficiently evacuated from desorbed water vapour together with the nitrogen carrier gas.

\subsection{A filter photometer}

A simpler instrument is the photometer design in Fig. 10. This is a small Cassegrain-type telescope with a channel electron multiplier (CEM) detector directly after the primary mirror instead of the secondary mirror. The detector here is a CEM with a $\mathrm{KBr}$ coated photocathode which has a spectral passband of $110-160 \mathrm{~nm}$. The absorption features of $\mathrm{O}_{2}$ can be used to obtain a filter that provides the EUV discrimination (Fig. 1). To this end, before the photons reach the channeltron, they pass through an $\mathrm{O}_{2}$ absorption cell enclosed between the channeltron and $\mathrm{agF}_{2}$ window. This 
results in a very narrow passband around the Lyman- $\alpha$ wavelength with high blocking of shorter and longer wavelengths up to about $180 \mathrm{~nm}$. The quantum efficiency of the $\mathrm{KBr}$ cathode is about $20 \%$ at $120 \mathrm{~nm}$. Electron multipliers with $\mathrm{O}_{2}$ filters have been utilised for measurements of Lyman- $\alpha$ from satellites. Examples are the MIO-1 and PIA-3 UV photometers onboard the Astrid-1 and Astrid-2 satellites respectively (Norberg et al., 1995; 2001), and the HDAC photometer on Cassini (Lauche and Zarnowiecki, 1992). As a simpler alternative to the $\mathrm{KBr}$ photocathode, also an uncoated CEM can be used. This provides a quantum efficiency of typically $1 \%$ up to $160-170 \mathrm{~nm}$ (Burle, 2006).

This photometer is a low mass and low cost alternative to the SLAM grating instrument. It features a higher throughput of the desired signal, good selectivity for the Lyman- $\alpha$ line, and good blocking of the shorter and longer wavelengths.

\section{Conclusions and outlook}

As shown in Fig. 2, the altitude range of NLC features strong vertical gradients in the direct solar Lyman- $\alpha$ during twilight conditions. For solar zenith angles larger than $90^{\circ}$, the diffuse geocoronal flux dominates over the direct flux at these altitudes. For quantitative studies of Lyman- $\alpha$ scattering by mesospheric ice particles, a well-defined source of the Lyman- $\alpha$ is important and, hence, the dominance of geocoronal contributions must be avoided. Therefore, the rocket-borne measurements described in this paper require experimental conditions with the sun significantly above the horizon.

As the Lyman- $\alpha$ flux has a strong control over ionospheric and chemical conditions in the mesosphere, this strong dependence on the solar zenith angle also has other consequences for studies of NLC and related phenomena. Lyman$\alpha$ gives rise to the ionospheric D-region by ionisation of NO. Lyman- $\alpha$ photolysis is also the major sink for water vapour. In addition, Murray and Plane (2005) have shown that the varying Lyman- $\alpha$ flux leads to substantial changes in the $\mathrm{O}_{\mathrm{x}} / \mathrm{HO}_{\mathrm{x}}$ chemistry in the vicinity of NLC between twilight and daytime. Hence, we expect significant differences in the charged and neutral environment of NLC when comparing twilight and daytime conditions. So far, the vast majority of rocket-borne studies of the polar summer mesosphere have been restricted to the twilight case. Hence, strong variability of the NLC environment due to Lyman- $\alpha$ radiation has not been taken into full consideration. For the future, complementary studies addressing mesopause conditions at smaller solar zenith angles are highly desirable.

A first flight opportunity for measuring Lyman- $\alpha$ in the vicinity of NLC was in July 2006 with SLAM as part of the eARI HotPay-1 sounding rocket from Norway. Under the 6th Framework Program of the European Union, the European Commission has promoted the utilization of the Arctic Lidar Observatory for Middle Atmosphere Research (ALOMAR) at Andøya Rocket Range, Norway. In particular, the EU contract was extended to the enhanced ALOMAR Research Infrastructure (eARI) including two sounding rocket launches in 2006 and 2007. For the first sounding rocket, HotPay1 , the atmospheric structure, composition and dynamics in the vicinity of NLC were the focus. The SLAM instrument was launched on HotPay-1 together with four other scientific instruments on 1 July 2006. While this test flight was intended to be the first measurement of the altitude- and angledependent scattering of Lyman- $\alpha$ by NLC in the mesosphere, a malfunction of the payload shortly after launch made it impossible to collect any mesospheric data.

A second flight opportunity for Lyman- $\alpha$ measurements is on the Swedish PHOCUS sounding rocket planned from Esrange, Sweden, in 2009. The major objective of PHOCUS (Particles, Hydrogen and Oxygen Chemistry in the Upper Summer mesosphere) is the interaction between NLC, meteoric particles and odd oxygen/odd hydrogen chemistry. In addition to a Lyman- $\alpha$ instrument, the PHOCUS payload will carry three photometers operating at two other wavelengths: 220 and $440 \mathrm{~nm}$. These will be mounted both forwardlooking $(220$ and $440 \mathrm{~nm})$ and tilted $(220 \mathrm{~nm})$ with respect to the payload axis. The forward looking photometers will be equipped with polarisers. Hence, PHOCUS aims at providing a complete in-situ optical characterisation of NLC particles, measuring colour ratios and phase functions as well as polarisation.

Acknowledgements. This work has been supported by the Swedish National Space Board. The HotPay-1 project was funded through the ALOMAR eARI initiative under the 6th Framework Programme of the European Union (contract number RITA-CT-2003-506208). J. Hedin thanks the Swedish National Graduate School of Space Technology for their support.

Edited by: U.-P. Hoppe

Reviewed by: E. Shettle and another anonymous referee

\section{References}

Alpers, M., Gerding, M., Höffner, J., and von Zahn, U.: NLC particle properties from a five-color lidar observation at $54^{\circ} \mathrm{N}, \mathrm{J}$. Geophys. Res., 105, 12 235-12 240, 2000.

Backhouse, T. W., The luminous cirrus cloud of June and July, Meteorol. Mag., 20, 133, 1885.

Bates, D. R. and Nicolet, M.: Atmospheric hydrogen, Planet. Space Sci., 13, 905-909, 1965.

Berger, U. and von Zahn, U.: Icy particles in the summer mesopause region: 3-d modelling of their environment and 2-d modelling of their transport, J. Geophys. Res., 107(A11), 1366, doi:10.1029/2001JA000316, 2002.

Bohren, C. F. and Huffman, D. R.: Absorption and Scattering of Light by Small Particles, John Wiley, New York, 1983.

Burle: Channeltron Electron Multiplier Handbook for Mass Spectrometry Applications, Burle Industries, Inc., Lancaster, USA, 2006. 
Carver, J. H., Gies, H. P., Hobbs, T. I., Lewis, B. R., and McCoy, D. G.: Temperature dependence of the molecular oxygen photoabsorption cross section near the Lyman $\alpha$ line, J. Geophys. Res., 82, 1955-1960, 1977.

DeLand, M. T., Shettle, E. P., Thomas, G. E., and Olivero, J. J.: Solar backscattered ultraviolet (SBUV) observations of polar mesopsheric clouds (PMCs) over two solar cycles, J. Geophys. Res., 108(D8), 8445, doi:10.1029/2002JD002398, 2003.

Eremenko, M. N., Petelina, S. V., Zasetsky, A. Y., Karlsson, B., Rinsland, C. P., Llewellyn, E. J., and Sloan, J. J.: Shape and composition of PMC particles derived from satellite remote sensing measurements. Geophys. Res. Lett., 32, L16S06, doi:10.1029/2005GL023013, 2005.

Friedlander, S. K.: Theoretical considerations for the particle size spectrum of the stratospheric aerosol, J. Meteor., 18, 753-759, 1961.

Goldberg, R., Kopp, E., and Witt, G.: An overview of NLC-91: A rocket/radar study of the polar summer mesosphere, Geophys. Res. Lett., 20, 2443-2446, 1993.

Goldberg, R. A., Pfaff, R. F., Holzworth, R. H., Schmidlin, F. J., Voss, H. D., Tuzzolino, A. J., Croskey, C. L., Mitchell, J. D., Friedrich, M., Murtagh, D., Witt, G., Gumbel, J., von Zahn, U., Singer, W., and Hoppe, U.-P.: DROPPS: A study of the polar summer mesosphere with rocket, radar and lidar, Geophys. Res. Lett., 28, 1407-1410, 2001.

Gumbel J., Stegman, J., Murtagh, D. P., and Witt, G.: Scattering phase functions and particle sizes in noctilucent clouds, Geophys. Res. Lett., 28, 1415-1418, 2001.

Gumbel, J. and Witt, G.: Rocket-borne photometry of NLC particle populations, Adv. Space Res, 28, 7, 1053-1058, 2001.

Hedin, A. E.: Extension of the MSIS thermosphere model into the middle and lower atmosphere, J. Geophys. Res., 96, 1159-1172, 1991.

Hervig, M., Thompson, R., McHugh, M., Gordley, L., Russel III, J., and Summers, M.: First confirmation that water ice is the primary component of polar mesospheric clouds, Geophys. Res. Lett., 28, 971-974. 2001.

Hunten, D. M., Turco, R. P., and Toon, O. B.: Smoke and dust particles of meteoric origin in the mesosphere and stratosphere, J. Atm. Sci., 37, 1342-1357, 1980.

Karlsson, B. and Rapp, M.: Latitudinal dependence of noctilucent cloud growth, Geophys. Res. Lett., 33, L11812, doi:10.1029/2006GL025805, 2006.

Kopp, E., Eberhardt, P., Herrmann, U., and Björn, L. G.: Positive ion composition of the high latitude D-region with noctilucent clouds, J. Geophys. Res., 90, 13 041-13 053, 1985.

Lauche, H. and Zarnowiecki, T.: The Lyman- $\alpha$ detector for Cassini mission, Proceedings from the 19th Annual European Meeting on Atmospheric Studies by Optical Methods, IRF Scientific Report 209, Swedish Institute of Space Physics, Kiruna, Sweden, 1992.

Lean, J.: Solar ultraviolet irradiance variations: A review, J. Geophys. Res., 92, 839-868, 1987.

Leslie, R.: Sky glows, Nature, 32, 245, 1885.

Lewis, B. R., Vardavas, I. M., and Carver, J. H.: The aeronomic dissociation of water vapor by solar H Lyman $\alpha$ radiation, J. Geophys. Res., 88, 4935-4940, 1983.

Lübken, F.-J.: Thermal structure of the Arctic summer mesosphere, J. Geophys. Res., 104, 9135-9149, 1999.
Meier, R. R.: Ultraviolet spectroscopy and remote sensing of the upper atmosphere, Space Sci. Rev., 59, 1-185, 1991.

Meier, R. R. and Mange, P.: Geocoronal hydrogen: An analysis of the Lyman-alpha airglow observed from OGO-4, Planet. Space Sci., 18, 803-821, 1970.

Meier, R. R. and Mange, P.: Spatial and temporal variations of the Lyman-alpha airglow and related atomic hydrogen distributions, Planet. Space Sci., 21, 309-327, 1973.

Merkel, A. W., Thomas, G. E., Palo, S. E., and Bailey, S. M.: Observations of the 5-day planetary wave in PMC measurements from the Student Nitric Oxide Explorer Satellite, Geophys. Res. Lett., 30(4), 1196, doi:10/1029/2002GL016524, 2003.

Murray, B. J. and Plane, J. M. C.: Modelling the impact of noctilucent cloud formation on atomic oxygen and other minor constituents of the summer mesosphere, Atmos. Chem. Phys., 5, 1027-1038, 2005, http://www.atmos-chem-phys.net/5/1027/2005/.

Norberg, O., Barabash, S., Sandahl, I., Lundin, R., Lauche, H., Koskinen, H., C:son Brandt, P., Roelof, E., Andersson, L., Eklund, U., Borg, H., Gimholt, J., Lundin, K., Rynö, J., and Olsen, S.: The Microsatellite Astrid, Proceedings of the 12th ESA Symposium on European Rocket and Balloon Programmes and Related Research, Lillehammer, Norway, 29 May-1 June, 1995.

Norberg, O., Winningham, J. D., Lauche, H., Keith, W., Puccio, W., Olsen, J., Lundin, K., and Scherrer, J.: The MEDUSA electron and ion spectrometer and the PIA ultraviolet photometers on Astrid-2, Ann. Geophys., 19, 593-600, 2001, http://www.ann-geophys.net/19/593/2001/.

Philbrick, C., Barnett, J., Gerndt, R., Offermann, D., Pendleton, W., Schlyter, P., Schmidlin, J., and Witt, G.: Temperature measurements during the CAMP program, Adv. Space Res., 4 (4), 153-156, 1984.

Plane, J. M. C.: The role of sodium bicarbonate in the nucleation of noctilucent clouds, Ann. Geophys, 18, 807-814, 2000

Prinz, D. K. and Brueckner, G. E.: Observations of the $\mathrm{O}_{2}$ column density between 120 and $70 \mathrm{~km}$ and absorption cross sections in the vicinity of H Lyman alpha, J. Geophys. Res., 82, 1481-1486, 1977.

Rapp, M., Lübken, F.-J., Müllemann, A., Thomas, G. E., and Jensen, E. J.: Small scale temperature variations in the vicinity of NLC: experimental and model results, J. Geophys. Res., 107 (D19), 4392, doi:10.1029/2001JD001241, 2002.

Rapp, M. and Thomas, G. E.: Modeling the microphysics of mesospheric ice particles: Assessment of current capabilities and basic sensitivities, J. Atm. Sol. Terr. Phys., 68, 715-744, 2006.

Rapp, M., Strelnikova, I., and Gumbel, J., Meteoric smoke particles: Evidence from rocket and radar techniques, Adv. Space. Res., 40, 809-817, 2007a.

Rapp, M., Thomas, G. E., and Baumgarten, G.: Spectral properties of mesospheric ice clouds: Evidence for non-spherical particles, J. Geophys. Res., 112, D03211, doi:10.1029/2006JD007322, $2007 b$.

Rosinski, J. and Snow, R. H.: Secondary particulate matter from meteor vapors, J. Meteorol. 18, 736-745, 1961.

Rusch, D. W., Thomas, G. E., and Jensen, E. J.: Particle size distributions in polar mesospheric clouds derived from solar mesosphere explorer measurements, J. Geophys. Res., 96, 12933 12939, 1991.

Seele, C. and Hartogh, P.: Water vapor of the polar middle atmo- 
sphere: annual variation and summer mesosphere conditions as observed by ground based microwave spectroscopy, Geophys. Res. Lett., 26, 1517-1520, 1999.

Thomas, G. E. and McKay, C. P.: On the mean particle size and water content of polar mesospheric clouds, Planet. Space Sci., 33, 1209-1224, 1985.

Thomas, G. E., Olivero, J. J., Jensen, E. J., Schröder, W., and Toon, O. B.: Relation between increasing methane and the presence of ice clouds at the mesopause, Nature, 338, 490-492, 1989.

Thrane, E. V. and Johannessen, A.: A measurement of the extinction of solar hydrogen Lyman- $\alpha$ radiation in the summer arctic mesosphere, J. Atm. Terr. Phys., 37, 655-661, 1975.

von Cossart, G., Fiedler, J., and von Zahn, U.: Size distributions of NLC particles as determined from 3-color observations of NLC by ground-based lidar, Geophys. Res. Lett., 26, 11, 1513-1516, 1999.

von Savigny, C., Petelina, S. V., Karlsson, B., Llewellyn, E. J., Degenstein, D. A., Loyd, N. D., and Burrows, J. P.: Vertical variation of NLC particle sizes retrieved from Odin/OSIRIS limb scattering observations, Geophys. Res. Lett., 32, L07806, doi:10.1029/2004GL021982, 2005.
Warren, S. G.: Optical constants of ice from the ultraviolet to the microwave, Appl. Optics, 23, 8, 1206-1225, 1984.

Witt, G., Martin-Löf, J., Wilhelm, N., and Smith, W. S.: High latitude summer mesospheric temperatures and winds with particular regard to noctilucent clouds, Space Research V, 820-821, 1964.

Witt, G.: Optical characteristics of mesospheric aerosol distributions in relation to noctilucent clouds, Tellus, 20, 1, 98-113, 1968.

Witt, G.: The nature of noctilucent clouds, Space Research IX, 157$169,1969$.

Witt, G., Dye, J. E., and Wilhelm, N.: Rocket-borne measurements of scattered sunlight in the mesopause, J. Atmos. Terr. Phys. 38, 223-238, 1975. 